\title{
Determining Optimal Parameters for Surface Roughness of Cylinder Liners During Honing Process by Taguchi Methodology - A Case Study
}

\author{
K. Prakash Babu \\ Assistant professor, Department of Mechanical Engineering \\ V.R Siddhartha Engineering College, Kanuru, Vijayawada, A.P-520 007. \\ M. Bala Chenniah \\ Assistant professor, Department of Mechanical Engineering \\ V.R Siddhartha Engineering College, Kanuru, Vijayawada, A.P-520 007. \\ A. Jagadeesh \\ UG Scholar, Department of Mechanical Engineering \\ V.R Siddhartha Engineering College, Kanuru, Vijayawada A.P-520 007.
}

\begin{abstract}
Honing is an abrasive machining process that produces a precision surface on a metal workpiece by scrubbing an abrasivestone against it along a controlled path. Honing is primarily used to improve the geometric form of a surface, but may also improve the surface texture. Typical applications are the finishing of cylinders for internal combustion engines, air bearing spindles and gears. In this present work we studied about the honing process used in the manufacturing of cylinder liners at Kusalava Internation Itd., Agiripalli and identified the various factors affecting the surface roughness of a cylinder liner during the process. By applying Taguchi methodology we determined the optimal parameters for obtaining a good surface finish for cylinder liners bore surface.
\end{abstract}

Key words: Honing, Taguchi methodology.

\section{INTRODUCTION}

Honing is a finishing process, in which a tool called hone carries out a combined rotary and reciprocating motion while the workpiece does not perform any working motion. Most honing is done on internal cylindrical surface, such as automobile cylindrical walls. The honing stones are held against the workpiece with controlled light pressure. The honing head is not guided externally but, instead, floats in the hole, being guided by the work surface. It is desired that Honing stones should not leave the work surface and Stroke length must cover the entire work length.

The honing stones are given a complex motion so as to prevent every single grit from repeating its path over the work surface. The critical process parameters are . Rotation speed, Oscillation speed, Length and position of the stroke and Honing stick pressure.

\section{IMPORTANCE OF SURFACE FINISH}

Cylinder liners are among the most critical engine components when it comes to oil consumption and frictional losses. Researchers have estimated that as much as $40 \%$ of the frictional losses in an engine arise from the friction between the cylinder liner and the piston ring. Therefore, high demands are set on the surface finish of the liner.

\section{NOMENCLATURE OF SURFACE ROUGHNESS}

In order to produce cylinder liners for engines with high demands regarding emissions and fuel consumption, the surface need to be characterized. There are multiple surface parameters that can be used to define the surface. Some of the most frequently used are the mean parameters. The most common one is the average roughness, $\mathrm{Ra}$. This is, as the name states, an average of the surface roughness over the sample length. 


$$
R a=\frac{1}{l} \int_{0}^{l}|Z(x)| d x \mid
$$

Where $(x)$ is the distance between the profile curve and the mean line and $l$ the sample length. Another parameter that is widely used in the industry is the $R q$ or RMS parameter. This is the root mean square of the surface roughness over the sample length and is calculated according to Equation

$$
R q=\frac{1}{l} \int_{0}^{l}\left|Z^{2}(x)\right| d x
$$

- Core roughness depth $\left(\boldsymbol{R}_{k}\right)$ : Depth of roughness core profile.

- Material portion (Mr1): Material portion, a level in percent (\%), determined from the intersection line that separates the protruding peaks from the roughness core profile.

- Material portion (Mr2): Material portion, a level in percent (\%), determined for the intersection line that separates the deep valleys from the roughness core profile.

- $\quad$ Reduced peak height $(\boldsymbol{R} p \boldsymbol{k})$ : Average height of the protruding peaks above the roughness core profile

- $\quad$ Reduced valley depths $(\boldsymbol{R} v \boldsymbol{k})$ : Average depth of the profile valleys projecting through the roughness core profile.

- Roughness average $(\boldsymbol{R} \boldsymbol{a})$ : The mean roughness is the arithmetic average of the absolute values of the roughness profile ordinates.

- Mean roughness depth $(\mathbf{R z})$ : The mean roughness depth is the arithmetic mean value of the single roughness depth of consecutive sampling lengths

- Maximum roughness (Rmax): The maximum roughness depth is the largest single roughness depth within the evaluation length

\section{TAGUCHI METHODOLOGY}

Genichi Taguchi has been identified with the advent of what has come to be termed quality engineering. The goal of quality engineering is to move quality improvement efforts upstream from the production phase to the product/process design stage.

\section{Steps Involved in Taguchi Method}

The use of Taguchi's parameter design involves the following steps

- Identify the main function and its side effects.

- Identify the noise factors, testing condition and quality characteristics.

- Identify the objective function to be optimized.

- Identify the control factors and their levels.

- Select a suitable Orthogonal Array and construct the Matrix

- Conduct the Matrix experiment.

- Examine the data; predict the optimum control factor levels and its performance.

- Conduct the verification experiment.

\section{Signal to Noise Ratios}

- In the parameter design stage Taguchi makes use of designed experiments and signal to noise ratios to determine the optimal parameter settings. The signal to noise ratios are derived from the Taguchi loss function. While Taguchi has proposed a large number of signal to noise ratios in which three are the most widely used.

$$
\begin{aligned}
& \text { Nominal is Best : } S N_{N}=10 \log \left(\frac{y^{-2}}{s^{2}}\right) \quad \text { Larger is Better }: S N_{L}=10 \log \left(\frac{\sum_{i=1}^{n} 1 / y_{i}^{2}}{n}\right) \\
& \text { Smaller is Better : } S N_{S}=10 \log \left(\frac{\sum_{i=1}^{n} y_{i}^{2}}{n}\right)
\end{aligned}
$$


Identify the main function and its side effects

\section{CASE STUDY}

Main function:Honing Operation on Cylinder Liner using Honing machine.

Side effects: Variation in surface finish.

Identify the noise factors, testing condition and quality characteristics

The "Factors" that affect honing operation on a machine are listed in the table below.

\begin{tabular}{|c|c|}
\hline Control parameters & Noise parameters \\
\hline Cutting Speed & Vibration \\
\hline Load & Raw material variation \\
\hline Number of strokes & Machine condition \\
\hline Noise radius & Temperature \\
\hline Coolant & Operator skill \\
\hline
\end{tabular}

Table-1

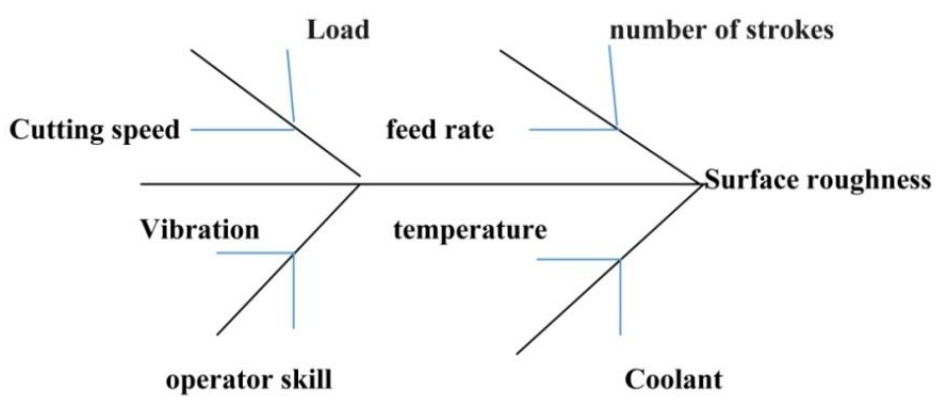

Fig.1 : Fish bone diagram representing the control and noise factors

\section{Identify the objective function to be optimized}

Objective Function: Smaller-the-Better

S/N Ratio for this function: $\eta=-10 \log _{10}\left(1 / \mathrm{n} \sum \mathrm{y}_{\mathrm{i}}{ }^{2}\right)$

Where, $n=$ Sample Size, and $y=$ Surface Roughness in that run.

\section{Identify the control factors and their levels}

The factors and their levels were decided for conducting the experiment, based on a "brain storming session" that was held with a group of people and also considering the guide lines given in the operator's manual provided by the manufacturer of the lathe machine. The factors and their levels are shown in table below.

\begin{tabular}{|c|c|c|c|}
\hline FACTORS & \multicolumn{3}{|c|}{ LEVELS } \\
\hline Cutting speed(rpm) & 115 & 125 & 140 \\
\hline Load for finish( kg/sq.cm) & 12 & 15 & 17 \\
\hline Number of strokes(stroke/piece) & 7 & 10 & 12 \\
\hline
\end{tabular}

Table-2 : Table with Selected Factors and their Levels

Select a suitable Orthogonal Array and construct the Matrix

If we consider three factors and three levels we should take L9 array as mentioned in Orthogonal array table below.

\begin{tabular}{|c|c|c|c|}
\hline Test case & Parameter1 & Parameter2 & Parameter3 \\
\hline 1 & 1 & 1 & 3 \\
\hline 2 & 1 & 2 & 2 \\
\hline 3 & 1 & 3 & 1 \\
\hline 4 & 2 & 1 & 2 \\
\hline 5 & 2 & 2 & 1 \\
\hline 6 & 2 & 3 & 3 \\
\hline 7 & 3 & 1 & 1 \\
\hline 8 & 3 & 2 & 3 \\
\hline 9 & 3 & 3 & 2 \\
\hline
\end{tabular}

Table-3: L9 orthogonal array

Volume 8 Issue 3 June 2017 


\section{CONDUCTING THE MATRIX EXPERIMENT}

In accordance with the above, experiments were conducted with their factors and their levels as mentioned in table. The experimental layout with the selected values of the factors is shown in Table. Each of the above 9 experiments were conducted 3 times (27 experiments in all) to account for the variations that may occur due to the noise factors. The surface roughness was measured using the surface roughness tester. The tables shows the measured values of surface roughness obtained from different experiments.

\begin{tabular}{|c|c|c|c|}
\hline \multirow{2}{*}{$\begin{array}{c}\text { Experiment } \\
\text { Number }\end{array}$} & \multicolumn{3}{|c|}{ Control Factors } \\
\cline { 2 - 4 } & Cutting Speed(rpm) & Load for Finish(kg/sq.cm) & No of Strokes(stroke/piece) \\
\hline 1 & 115 & 12 & 7 \\
\hline 2 & 115 & 15 & 10 \\
\hline 3 & 115 & 17 & 10 \\
\hline 4 & 125 & 12 & 7 \\
\hline 5 & 125 & 15 & 12 \\
\hline 6 & 125 & 17 & 7 \\
\hline 7 & 140 & 12 & 12 \\
\hline 8 & 140 & 15 & 10 \\
\hline 9 & 140 & 17 & 7 \\
\hline
\end{tabular}

Table-4

\begin{tabular}{|c|c|c|c|c|c|}
\hline $\begin{array}{c}\text { EXP } \\
\text { NO }\end{array}$ & $\begin{array}{c}\text { PARA } \\
\text { METER }\end{array}$ & $\begin{array}{c}\text { TRAIL } \\
\mathbf{1}\end{array}$ & $\begin{array}{c}\text { TRAIL } \\
\mathbf{2}\end{array}$ & $\begin{array}{c}\text { TRAIL } \\
\mathbf{3}\end{array}$ & MEAN \\
\hline 1 & $\mathbf{R a}$ & 0.4375 & 0.5662 & 0.3873 & 0.4636 \\
\hline & $\mathbf{R z}$ & 3.89 & 4.27 & 3.66 & 3.94 \\
\hline & $\mathbf{R m a x}$ & 6.22 & 5.94 & 5.22 & 5.79 \\
\hline & $\mathbf{R k}$ & 0.811 & 1.093 & 1.041 & 0.981 \\
\hline & $\mathbf{R p k}$ & 0.2811 & 0.2522 & 0.3182 & 0.2838 \\
\hline & $\mathbf{R v k}$ & 1.64 & 1.83 & 1.55 & 1.67 \\
\hline & $\mathbf{M r 1}$ & 7.4 & 5.83 & 7.23 & 6.82 \\
\hline & $\mathbf{M r 2}$ & 78.9 & 75.7 & 79.6 & 78.06 \\
\hline
\end{tabular}

\begin{tabular}{|c|c|c|c|c|c|}
\hline $\begin{array}{c}\text { EXP } \\
\text { NO }\end{array}$ & $\begin{array}{c}\text { PARA } \\
\text { METER }\end{array}$ & $\begin{array}{c}\text { TRAIL } \\
\mathbf{1}\end{array}$ & $\begin{array}{c}\text { TRAIL } \\
\mathbf{2}\end{array}$ & $\begin{array}{c}\text { TRAIL } \\
\mathbf{3}\end{array}$ & MEAN \\
\hline 2 & $\mathbf{R a}$ & 0.5133 & 0.5945 & 0.4801 & 0.5293 \\
\hline & $\mathbf{R z}$ & 3.97 & 4.69 & 4.08 & 4.25 \\
\hline & $\mathbf{R m a x}$ & 6.49 & 6.36 & 6 & 6.28 \\
\hline & $\mathbf{R k}$ & 0.729 & 1.328 & 0.754 & 0.937 \\
\hline & $\mathbf{R p k}$ & 0.2530 & 0.3350 & 0.323 & 0.2736 \\
\hline & $\mathbf{R v k}$ & 1.49 & 2.00 & 1.68 & 1.72 \\
\hline & Mr1 & 7.87 & 6.21 & 7.06 & 7.04 \\
\hline & $\mathbf{M r 2}$ & 81.9 & 78.6 & 78.4 & 79.6 \\
\hline
\end{tabular}

\begin{tabular}{|c|c|c|c|c|c|}
\hline $\begin{array}{c}\text { EXP } \\
\text { NO }\end{array}$ & $\begin{array}{c}\text { PARA } \\
\text { METER }\end{array}$ & $\begin{array}{c}\text { TRAIL } \\
\mathbf{1}\end{array}$ & $\begin{array}{c}\text { TRAIL } \\
\mathbf{2}\end{array}$ & $\begin{array}{c}\text { TRAIL } \\
\mathbf{3}\end{array}$ & MEAN \\
\hline 3 & $\mathbf{R a}$ & 0.4166 & 0.3456 & 0.3850 & 0.3824 \\
\hline & $\mathbf{R z}$ & 3.86 & 3.48 & 3.56 & 3.63 \\
\hline & $\mathbf{R m a x}$ & 5.28 & 5.03 & 5.20 & 5.17 \\
\hline & $\mathbf{R k}$ & 0.927 & 0.766 & 0.759 & 0.817 \\
\hline & $\mathbf{R p k}$ & 0.2645 & 0.2213 & 0.2118 & 0.2325 \\
\hline & $\mathbf{R v k}$ & 1.56 & 1.35 & 1.11 & 1.34 \\
\hline & Mr1 & 6.98 & 6.88 & 6.63 & 6.83 \\
\hline & $\mathbf{M r 2}$ & 79.2 & 80.9 & 81.1 & 80.4 \\
\hline
\end{tabular}

\begin{tabular}{|c|c|c|c|c|c|}
\hline $\begin{array}{c}\text { EXP } \\
\text { NO }\end{array}$ & $\begin{array}{c}\text { PARA } \\
\text { METER }\end{array}$ & $\begin{array}{c}\text { TRAIL } \\
\mathbf{1}\end{array}$ & $\begin{array}{c}\text { TRAIL } \\
\mathbf{2}\end{array}$ & $\begin{array}{c}\text { TRAIL } \\
\mathbf{3}\end{array}$ & MEAN \\
\hline 4 & $\mathbf{R a}$ & 0.4413 & 0.5325 & 0.4847 & 0.4861 \\
\hline & $\mathbf{R z}$ & 4.09 & 4.85 & 4.11 & 4.35 \\
\hline & $\mathbf{R m a x}$ & 6.37 & 7.36 & 6.48 & 6.73 \\
\hline & $\mathbf{R k}$ & 1.446 & 0.996 & 1.32 & 1.25 \\
\hline & $\mathbf{R p k}$ & 0.407 & 0.313 & 0.337 & 0.352 \\
\hline & $\mathbf{R v k}$ & 1.84 & 2 & 10.9 & 1.91 \\
\hline & Mr1 & 7.11 & 6.91 & 6.24 & 6.75 \\
\hline & $\mathbf{M r 2}$ & 80.5 & 78.3 & 79.08 & 79.29 \\
\hline
\end{tabular}

\begin{tabular}{|c|c|c|c|c|c|}
\hline $\begin{array}{c}\text { EXP } \\
\text { NO }\end{array}$ & $\begin{array}{c}\text { PARA } \\
\text { METER }\end{array}$ & $\begin{array}{c}\text { TRAIL } \\
\mathbf{1}\end{array}$ & $\begin{array}{c}\text { TRAIL } \\
\mathbf{2}\end{array}$ & $\begin{array}{c}\text { TRAIL } \\
\mathbf{3}\end{array}$ & MEAN \\
\hline 5 & $\mathbf{R a}$ & 0.4675 & 0.5245 & 0.5841 & 0.5253 \\
\hline & $\mathbf{R z}$ & 4.41 & 4.59 & 5.34 & 4.79 \\
\hline & $\mathbf{R m a x}$ & 7.40 & 6.22 & 7.78 & 7.12 \\
\hline & $\mathbf{R k}$ & 0.9296 & 0.686 & 0.704 & 0.772 \\
\hline & $\mathbf{R p k}$ & 0.269 & 0.36 & 0.38 & 0.336 \\
\hline & $\mathbf{R v k}$ & 1.7 & 1.8 & 2.12 & 1.87 \\
\hline & $\mathbf{M r 1}$ & 7.07 & 6.53 & 6.42 & 6.88 \\
\hline & $\mathbf{M r 2}$ & 78.7 & 79.86 & 78.52 & 78.02 \\
\hline
\end{tabular}

\begin{tabular}{|c|c|c|c|c|c|}
\hline $\begin{array}{c}\text { EXP } \\
\text { NO }\end{array}$ & $\begin{array}{c}\text { PARA } \\
\text { METER }\end{array}$ & $\begin{array}{c}\text { TRAIL } \\
\mathbf{1}\end{array}$ & $\begin{array}{c}\text { TRAIL } \\
\mathbf{2}\end{array}$ & $\begin{array}{c}\text { TRAIL } \\
\mathbf{3}\end{array}$ & MEAN \\
\hline 6 & $\mathbf{R a}$ & 0.5321 & 0.4706 & 0.4422 & 0.4483 \\
\hline & $\mathbf{R z}$ & 4.19 & 6.38 & 4.22 & 4.93 \\
\hline & $\mathbf{R m a x}$ & 5.20 & 5.63 & 6.74 & 5.85 \\
\hline & $\mathbf{R k}$ & 0.773 & 0.992 & 0.694 & 0.819 \\
\hline & $\mathbf{R p k}$ & 0.227 & 0.424 & 0.295 & 0.315 \\
\hline & $\mathbf{R v k}$ & 1.70 & 1.43 & 1.89 & 1.67 \\
\hline & Mr1 & 6.41 & 7.02 & 7.03 & 6.82 \\
\hline & $\mathbf{M r 2}$ & 79.86 & 77.07 & 77.87 & 78.26 \\
\hline
\end{tabular}

\begin{tabular}{|c|c|c|c|c|c|}
\hline $\begin{array}{c}\text { EXP } \\
\text { NO }\end{array}$ & $\begin{array}{c}\text { PARA } \\
\text { METER }\end{array}$ & $\begin{array}{c}\text { TRAIL } \\
\mathbf{1}\end{array}$ & $\begin{array}{c}\text { TRAIL } \\
\mathbf{2}\end{array}$ & $\begin{array}{c}\text { TRAIL } \\
\mathbf{3}\end{array}$ & MEAN \\
\hline 7 & $\mathbf{R a}$ & 0.4501 & 0.3638 & 0.4793 & 0.4310 \\
\hline & $\mathbf{R z}$ & 4.91 & 4.50 & 3.58 & 4.33 \\
\hline & $\mathbf{R m a x}$ & 10.18 & 7.70 & 6.38 & 8.08 \\
\hline & $\mathbf{R k}$ & 0.895 & 1.181 & 0.832 & 0.969 \\
\hline & Rpk & 0.344 & 0.357 & 0.272 & 0.324 \\
\hline & $\mathbf{R v k}$ & 1.71 & 1.68 & 1.36 & 1.58 \\
\hline & $\mathbf{M r 1}$ & 7.79 & 7.96 & 6.58 & 7.41 \\
\hline & $\mathbf{M r 2}$ & 79.51 & 78.56 & 81.07 & 79.79 \\
\hline
\end{tabular}

\begin{tabular}{|c|c|c|c|c|c|}
\hline $\begin{array}{c}\text { EXP } \\
\text { NO }\end{array}$ & $\begin{array}{c}\text { PARA } \\
\text { METER }\end{array}$ & $\begin{array}{c}\text { TRAIL } \\
\mathbf{1}\end{array}$ & $\begin{array}{c}\text { TRAIL } \\
\mathbf{2}\end{array}$ & $\begin{array}{c}\text { TRAIL } \\
\mathbf{3}\end{array}$ & MEAN \\
\hline 8 & $\mathbf{R a}$ & 0.4375 & 0.5945 & 0.3873 & 0.4731 \\
\hline & $\mathbf{R z}$ & 4.91 & 4.41 & 4.09 & 4.47 \\
\hline & $\mathbf{R m a x}$ & 6.22 & 6.49 & 5.28 & 5.99 \\
\hline & $\mathbf{R k}$ & 0.737 & 0.686 & 0.740 & 0.721 \\
\hline & Rpk & 0.225 & 0.300 & 0.289 & 0.271 \\
\hline & $\mathbf{R v k}$ & 1.70 & 1.89 & 2.00 & 1.86 \\
\hline & $\mathbf{M r 1}$ & 8.81 & 6.20 & 8.89 & 7.96 \\
\hline & $\mathbf{M r 2}$ & 79.36 & 80.40 & 80.10 & 79.95 \\
\hline
\end{tabular}

\begin{tabular}{|c|c|c|c|c|c|}
\hline $\begin{array}{c}\text { EXP } \\
\text { NO }\end{array}$ & PARAMETER & TRAIL & TRAIL & TRAIL & MEAN \\
\hline
\end{tabular}

Volume 8 Issue 3 June 2017 


\begin{tabular}{|c|c|c|c|c|c|}
\hline 9 & Ra & 0.4166 & 0.5325 & 0.3850 & 0.4447 \\
\hline & $\mathbf{R z}$ & 6.38 & 4.59 & 4.85 & 5.27 \\
\hline & Rmax & 5.49 & 6.00 & 5.03 & 5.50 \\
\hline & $\mathbf{R k}$ & 1.110 & 0.874 & 0.825 & 0.936 \\
\hline & $\mathbf{R p k}$ & 0.301 & 0.352 & 0.256 & 0.303 \\
\hline & $\mathbf{R v k}$ & 1.65 & 1.30 & 1.62 & 1.52 \\
\hline & $\mathbf{M r 1}$ & 7.39 & 5.83 & 6.76 & 6.66 \\
\hline & $\mathbf{M r 2}$ & 76.80 & 80.46 & 77.17 & 78.14 \\
\hline
\end{tabular}

Tables-5: Measured values of surface Roughness by Experimentations

\section{Examination of Data of Ra}

The following are the experimental results of the work carried out.

\section{Experimental Details}

Since the objective function (Surface Finish) is smaller-the-better type of control function, was used in

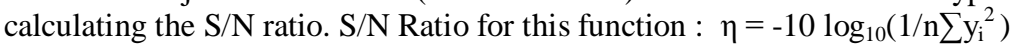

The $\mathrm{S} / \mathrm{N}$ ratios of all the experiments were calculated and tabulated as shown in Table

\begin{tabular}{|c|c|c|c|c|c|c|c|c|c|}
\hline Exp No. & 1 & 2 & 3 & 4 & 5 & 6 & 7 & 8 & 9 \\
\hline S/N Ratio & 6.562 & 5.49 & 8.324 & 6.238 & 5.555 & 6.319 & 7.254 & 6.3523 & 6.951 \\
\hline
\end{tabular}

\section{Table-6}

The S/N ratio for the individual control factors are calculated as given below:
$\mathrm{S}_{\mathrm{s} 1}=\eta_{1}+\eta_{2}+\eta_{3}$
$\mathrm{S}_{\mathrm{L} 1}=\eta_{1}+\eta_{4}+\eta_{7}$
$\mathrm{S}_{\mathrm{N} 1}=\eta_{1}+\eta_{5}+\eta_{9}$
$\mathrm{S}_{\mathrm{s} 2}=\eta_{7}+\eta_{8}+\eta_{9}$
$\mathrm{S}_{\mathrm{L} 2}=\eta_{2}+\eta_{5}+\eta_{8}$
$S_{\mathrm{N} 2}=\eta_{2}+\eta_{6}+\eta_{7}$
$\mathrm{S}_{\mathrm{s} 3}=\eta_{7}+\eta_{8}+\eta_{9}$
$\mathrm{S}_{\mathrm{L} 3}=\eta_{3}+\eta_{6}+\eta_{7}$
$\mathrm{S}_{\mathrm{N} 3}=\eta_{3}+\eta_{4}+\eta_{8}$

For selecting the values of $\eta_{1}, \eta 2, \eta 3$ etc. and to calculate Ss1, Ss2 \& Ss3 see table.

$\eta \mathrm{k}$ is the $\mathrm{S} / \mathrm{N}$ ratio corresponding to Experiment $\mathrm{k}$.

- Average S/N ratio corresponding to Cutting Speed at level $1=\mathrm{Ss} 1 / 3$

- Average S/N ratio corresponding to Cutting Speed at level $2=\mathrm{Ss} 2 / 3$

- Average $\mathrm{S} / \mathrm{N}$ ratio corresponding to Cutting Speed at level $3=\mathrm{Ss} 3 / 3$

$\mathrm{j}$ is the corresponding level each factor. Similarly Sfj and Stj are calculated for feed and depth of cut. The average of the signal to noise ratios is shown in table. Similarly $\mathrm{S} / \mathrm{N}$ ratios can be calculated for other factors.

\begin{tabular}{|c|c|c|c|c|c|c|}
\hline \multirow{2}{*}{$\begin{array}{c}\text { Level } \\
\text { Ra }\end{array}$} & \multicolumn{2}{|c|}{ Cutting Speed } & \multicolumn{2}{c|}{ Load for Finish } & \multicolumn{2}{c|}{ No of Strokes } \\
\cline { 2 - 7 } & $\begin{array}{c}\text { Sum } \\
(\mathrm{Ss})\end{array}$ & $\begin{array}{c}\text { Avg } \\
\text { S/Nratio }\end{array}$ & Sum(S1) & $\begin{array}{c}\text { Avg } \\
\text { S/Nratio }\end{array}$ & Sum(Sn) & $\begin{array}{c}\text { Avg } \\
\text { S/Nratio }\end{array}$ \\
\hline 1 & 20.376 & 6.792 & 20.054 & 6.684 & 19.068 & 6.356 \\
\hline 2 & 18.112 & 6.037 & 17.397 & 5.799 & 19.063 & 6.354 \\
\hline 3 & 20.557 & 6.852 & 21.594 & 7.918 & 20.914 & 6.971 \\
\hline
\end{tabular}

Table-7

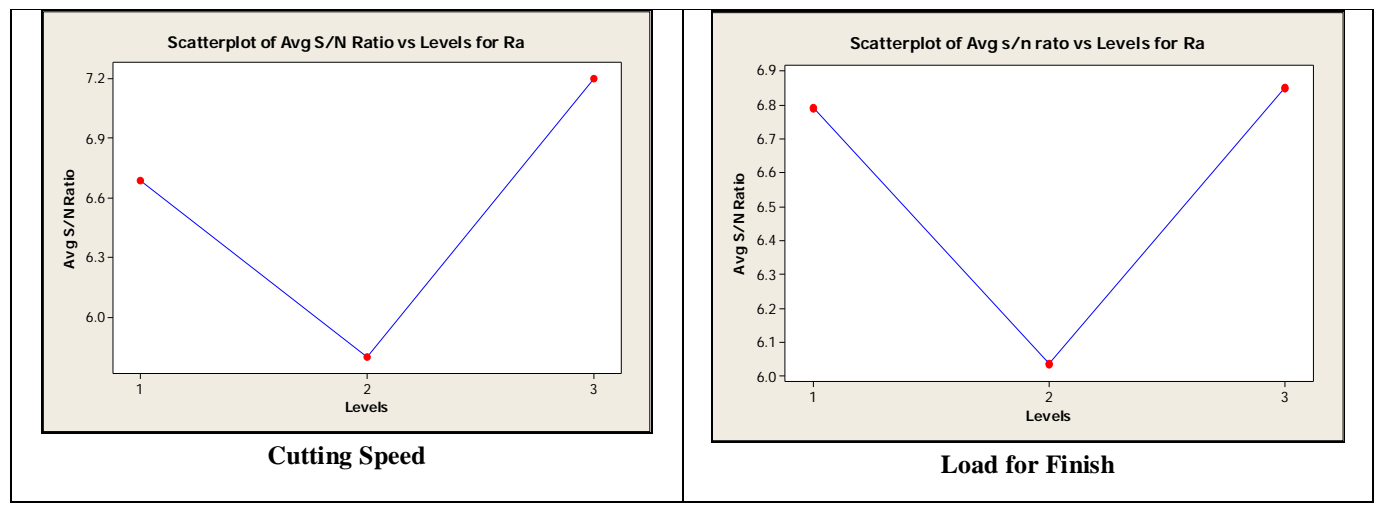




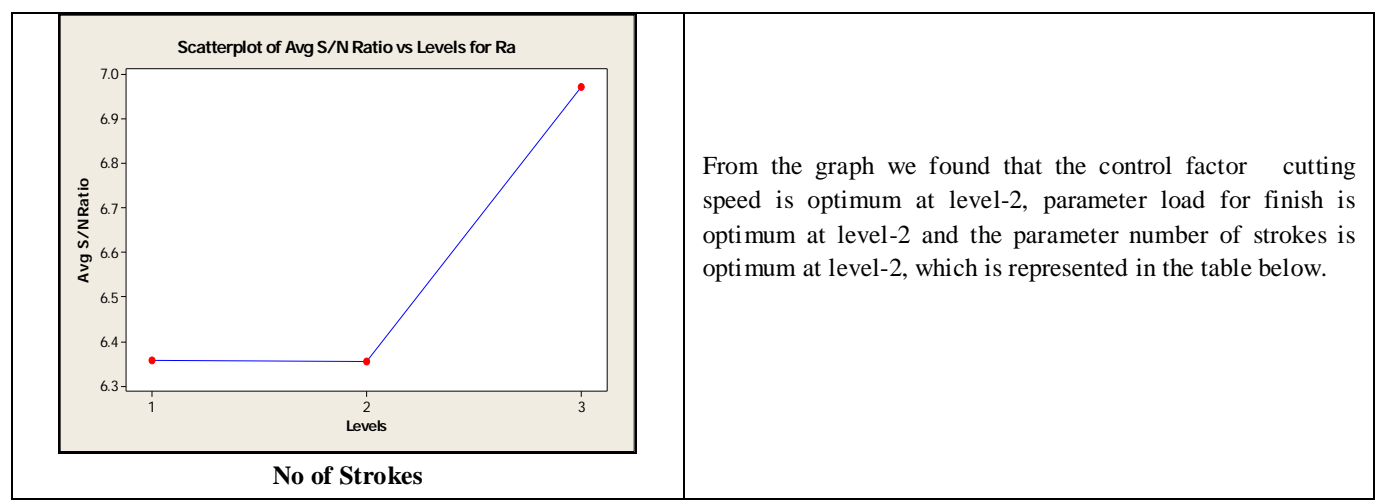

Fig.2 : Graphical representation of Avg S/N ratio vs Parameter Levels Optimum values of factors and their corresponding levels

\begin{tabular}{|c|c|}
\hline Factors & Optimum Value \\
\hline Cutting Speed(rpm) & 125 \\
\hline Load for Finish(kg/sq.cm) & 15 \\
\hline No of Strokes(stroke/piece) & 10 \\
\hline
\end{tabular}

Table-8

\section{Examination of Data of Rk}

The following are the experimental results of the work carried out.

\section{Experimental Details}

Since the objective function (Surface Finish) is smaller-the-better type of control function, was used in calculating the $\mathrm{S} / \mathrm{N}$ ratio. $\mathrm{S} / \mathrm{N}$ Ratio for this function : $\eta=-10 \log _{10}\left(1 / \mathrm{n} \sum \mathrm{y}_{\mathrm{i}}^{2}\right)$

The S/N ratios of all the experiments were calculated and tabulated as shown in Table

\begin{tabular}{|c|c|c|c|c|c|c|c|c|c|}
\hline Exp No. & 1 & 2 & 3 & 4 & 5 & 6 & 7 & 8 & 9 \\
\hline S/N Ratio & 0.093 & 0.202 & 1.713 & -2.064 & 2.162 & 1.626 & 0.165 & 2.836 & 0.495 \\
\hline
\end{tabular}

Table-9

\begin{tabular}{|c|c|c|c|c|c|c|}
\hline \multirow{2}{*}{$\begin{array}{c}\text { Level } \\
\text { Rk }\end{array}$} & \multicolumn{2}{|c|}{ Cutting Speed } & \multicolumn{2}{c|}{ Load for Finish } & \multicolumn{2}{c|}{ No of Strokes } \\
\cline { 2 - 7 } & $\begin{array}{c}\text { Sum } \\
\text { (Ss) }\end{array}$ & $\begin{array}{c}\text { Avg } \\
\text { S/Nratio }\end{array}$ & Sum(S1) & $\begin{array}{c}\text { Avg } \\
\text { S/Nratio }\end{array}$ & Sum(Sn) & $\begin{array}{c}\text { Avg } \\
\text { S/Nratio }\end{array}$ \\
\hline 1 & 2.008 & 0.669 & -1.806 & -0.602 & 2.75 & 0.917 \\
\hline 2 & 1.724 & 0.575 & 5.2 & 1.733 & 1.993 & 0.664 \\
\hline 3 & 3.496 & 1.165 & 3.83 & 1.27 & 2.48 & 0.82 \\
\hline
\end{tabular}

Table-10: Average of the signal to noise ratios

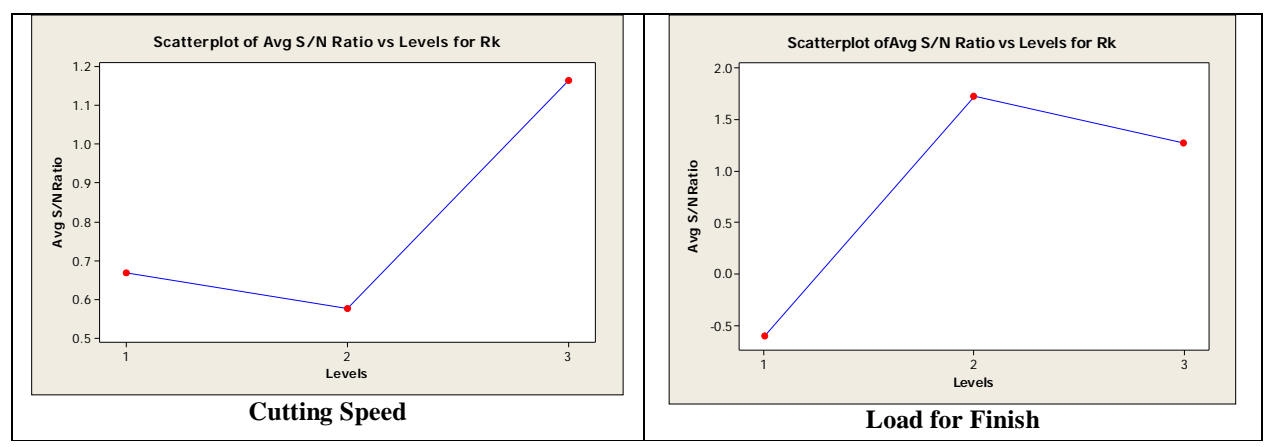




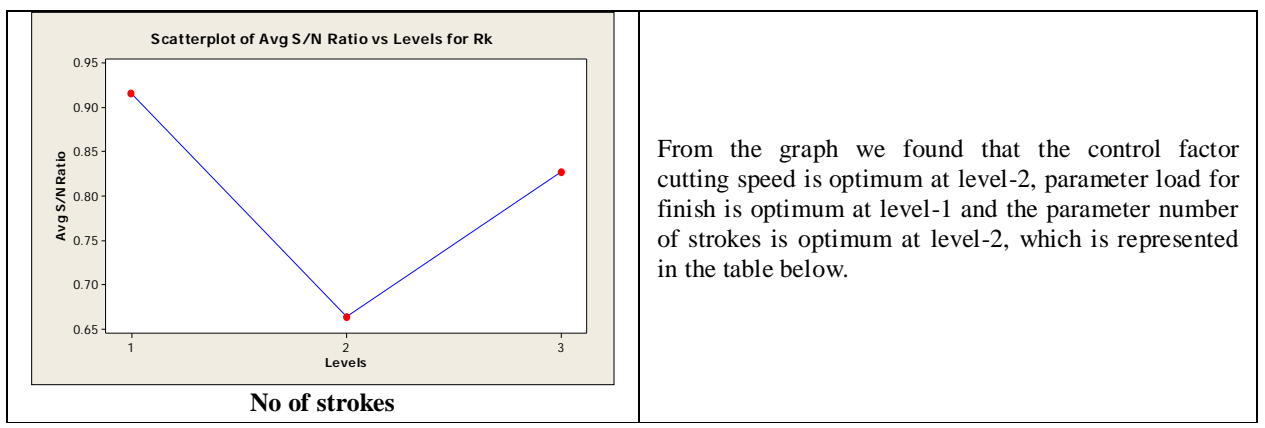

Fig.3: Graphical representation of Avg S/N ratio vs Parameter Levels

Optimum values of factors and their levels

\begin{tabular}{|c|c|}
\hline Parameter & Optimum Value \\
\hline Cutting Speed(rpm) & 125 \\
\hline Load for Finish(kg/sq.cm) & 12 \\
\hline No of Strokes(strokes/piece) & 10 \\
\hline
\end{tabular}

Table-11

\section{Examination of Data of RPk}

The following are the experimental results of the work carried out.

\section{Experimental Details}

Since the objective function (Surface Finish) is smaller-the-better type of control function, was used in calculating the $\mathrm{S} / \mathrm{N}$ ratio. $\mathrm{S} / \mathrm{N}$ Ratio for this function : $\eta=-10 \log _{10}\left(1 / \mathrm{n} \sum \mathrm{y}_{\mathrm{i}}^{2}\right)$

The $\mathrm{S} / \mathrm{N}$ ratios of all the experiments were calculated and tabulated as shown in Table

\begin{tabular}{|c|c|c|c|c|c|c|c|c|c|}
\hline Exp No. & 1 & 2 & 3 & 4 & 5 & 6 & 7 & 8 \\
\hline S/N Ratio & 10.899 & 10.291 & 12.628 & 9.005 & 9.376 & 9.742 & 9.723 & 11.266 & 10.299 \\
\hline
\end{tabular}

Table-12

\begin{tabular}{|c|c|c|c|c|c|c|}
\hline \multirow{2}{*}{$\begin{array}{c}\text { Level } \\
\text { Rpk }\end{array}$} & \multicolumn{2}{|c|}{ Cutting Speed } & \multicolumn{2}{c|}{ Load for Finish } & \multicolumn{2}{c|}{ No of Strokes } \\
\cline { 2 - 7 } & $\begin{array}{c}\text { Sum } \\
\text { (Ss) }\end{array}$ & $\begin{array}{c}\text { Avg } \\
\text { S/Nratio }\end{array}$ & Sum(S1) & $\begin{array}{c}\text { Avg } \\
\text { S/Nratio }\end{array}$ & Sum(Sn) & $\begin{array}{c}\text { Avg } \\
\text { S/Nratio }\end{array}$ \\
\hline 1 & 33.81 & 11.27 & 29.62 & 9.87 & 30.57 & 10.19 \\
\hline 2 & 28.12 & 9.37 & 30.93 & 10.31 & 29.75 & 9.91 \\
\hline 3 & 31.28 & 10.42 & 32.66 & 10.88 & 32.9 & 10.96 \\
\hline
\end{tabular}

Table-13: The average of the signal to noise ratios

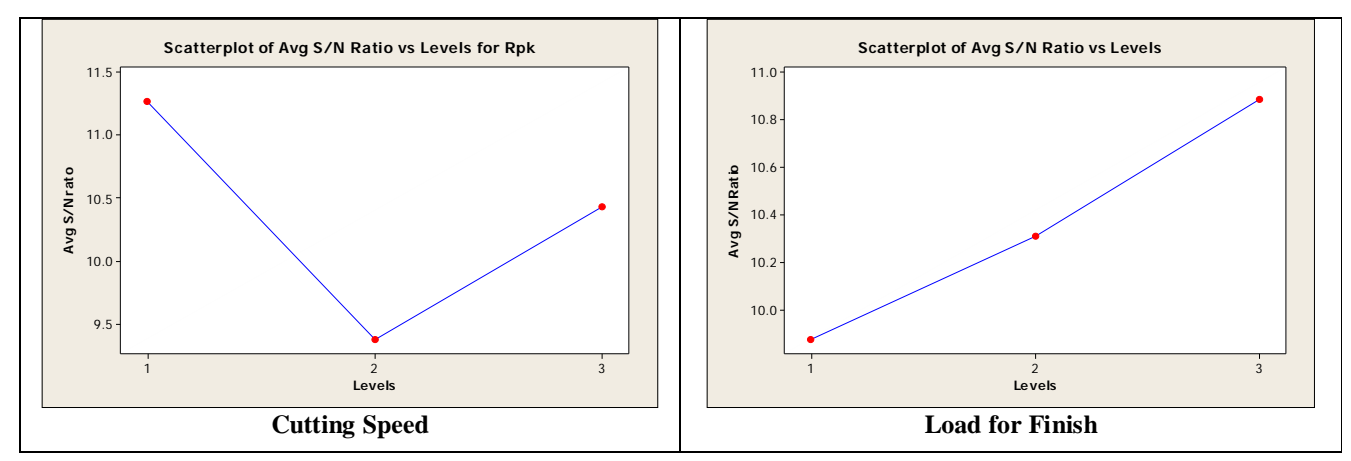




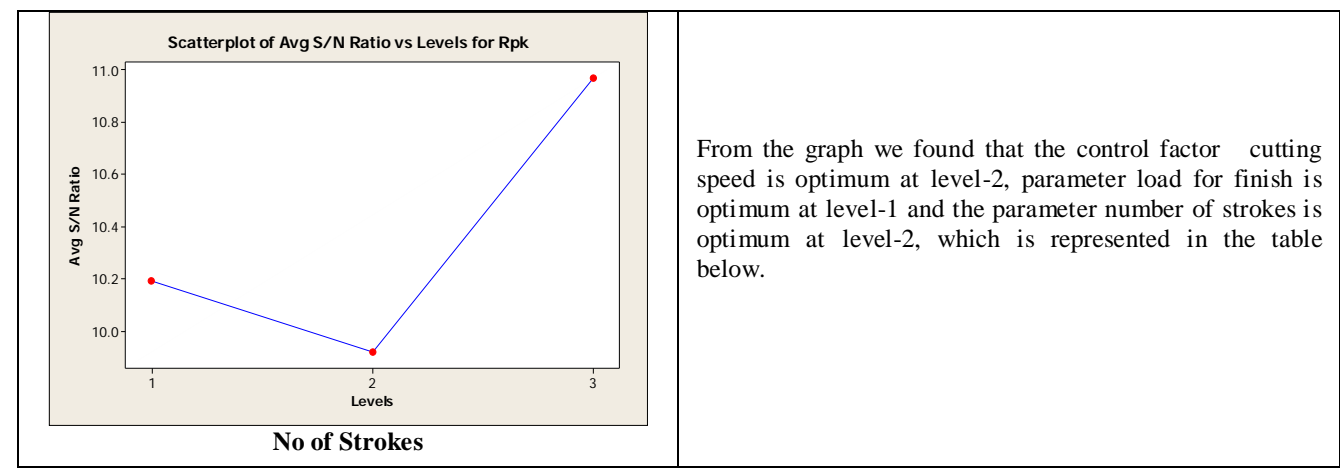

Fig.4: Graphical representation of Avg S/N ratio vs Parameter Levels

Optimum values of factors and their levels

\begin{tabular}{|c|c|}
\hline Parameter & Optimum Value \\
\hline Cutting Speed(rpm) & 125 \\
\hline Load for Finish(kg/sq.cm) & 12 \\
\hline No of Strokes(strokes/piece) & 10 \\
\hline
\end{tabular}

Table-14

\section{RESULTS \& DISCUSSIONS}

In the present work nine experiments each three trails were carried out and optimal parameters for the eight surface roughness characteristics were determined separately. from the above calculations and their corresponding graphs we observed that most of them are indicating level II for cutting speed, level I for load for finish and level II for no of strokes which are indicated in the below table.

\section{Optimum values of factors and their levels}

\begin{tabular}{|c|c|}
\hline Parameter & Optimum Value \\
\hline Cutting Speed(rpm) & 125 \\
\hline Load for Finish(kg/sq.cm) & 12 \\
\hline No of Strokes(strokes/piece) & 10 \\
\hline
\end{tabular}

Table-15

\section{CONFIRMATION TRAIL/VERIFICATION EXPERIMENTATION}

\begin{tabular}{|c|c|c|c|c|c|}
\hline EXP & \multirow{2}{*}{ PARAMETER } & TRAIL & TRAIL & TRAIL & \multirow{2}{*}{ MEAN } \\
\hline & Ra & 0.4166 & 0.3456 & 0.3850 & 0.3824 \\
\hline & Rz & 3.86 & 3.48 & 3.56 & 3.63 \\
\hline & Rmax & 5.28 & 5.03 & 5.20 & 5.17 \\
\hline & Rk & 0.927 & 0.766 & 0.759 & 0.817 \\
\hline & Rpk & 0.2645 & 0.2213 & 0.2118 & 0.2325 \\
\hline & Rvk & 1.56 & 1.35 & 1.11 & 1.34 \\
\hline & Mr1 & 6.98 & 6.88 & 6.63 & 6.83 \\
\hline & Mr2 & 79.2 & 80.9 & 81.1 & 80.4 \\
\hline
\end{tabular}

Table-16: Measured Surface Roughness Values

\begin{tabular}{|c|c|c|c|c|c|c|c|c|c|}
\hline Exp No. & 1 & 2 & 3 & 4 & 5 & 6 & 7 & 8 & 9 \\
\hline S/N Ratio & 10.899 & 10.291 & 12.628 & 9.005 & 9.376 & 9.742 & 9.723 & 11.266 & 10.299 \\
\hline
\end{tabular}

Table-17: The $\mathrm{S} / \mathrm{N}$ ratios of all the experiments were calculated and tabulated

\begin{tabular}{|c|c|c|c|c|c|c|}
\hline \multirow{3}{*}{ Level } & \multicolumn{2}{|c|}{ Cutting Speed } & \multicolumn{2}{c|}{ Load for Finish } & \multicolumn{2}{c|}{ No of Strokes } \\
\cline { 2 - 7 } & $\begin{array}{c}\text { Sum } \\
\text { (Ss) }\end{array}$ & $\begin{array}{c}\text { Avg } \\
\text { S/Nratio }\end{array}$ & Sum(S1) & $\begin{array}{c}\text { Avg } \\
\text { S/Nratio }\end{array}$ & Sum(Sn) & $\begin{array}{c}\text { Avg } \\
\text { S/Nratio }\end{array}$ \\
\hline 1 & 33.81 & 11.27 & 29.62 & 9.87 & 30.57 & 10.19 \\
\hline 2 & 28.12 & 9.37 & 30.93 & 10.31 & 29.75 & 9.91 \\
\hline
\end{tabular}

Volume 8 Issue 3 June 2017 


\begin{tabular}{|l|l|l|l|l|l|l|}
\hline 3 & 31.28 & 10.42 & 32.66 & 10.88 & 32.9 & 10.96 \\
\hline
\end{tabular}

\section{Table-18: The average of the signal to noise ratios}

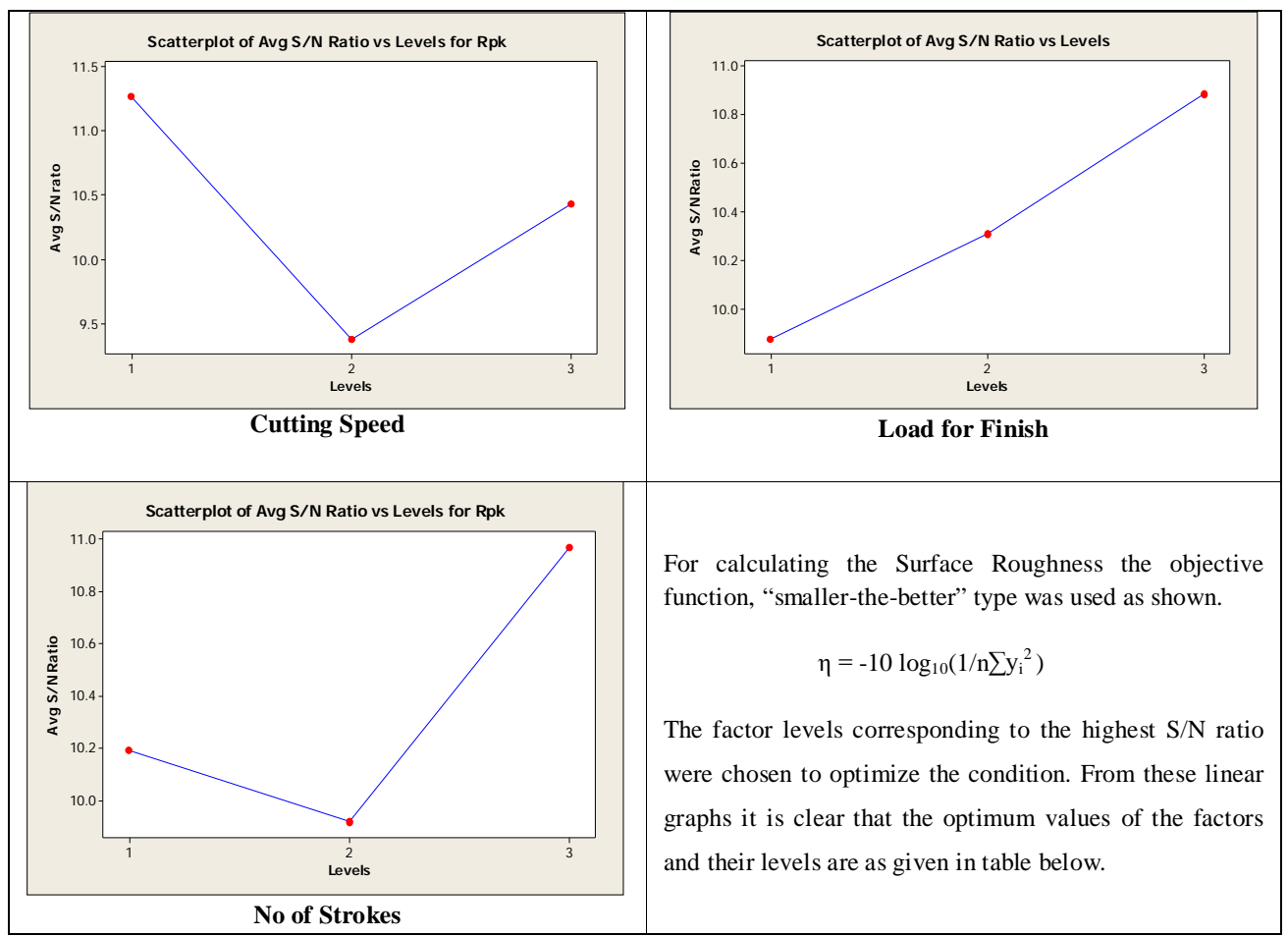

Fig.5: Graphical representation of Avg S/N ratio vs Parameter Levels:

\section{Optimum values of factors and their levels}

\begin{tabular}{|c|c|}
\hline Parameter & Optimum Value \\
\hline Cutting Speed(rpm) & 125 \\
\hline Load for Finish(kg/sq.cm) & 12 \\
\hline No of Strokes(strokes/piece) & 10 \\
\hline
\end{tabular}

Table-19

Hence verification experiment has been done successfully.

\section{CONCLUSION}

In the present work nine experiments each three trails were carried out and optimal parameters for the eight surface roughness characteristics were determined separately. But due to space limitation only few important characteristics and their respective calculations are presented in this paper. From the above calculations and their corresponding graphs we observed that most of them are indicating level II for cutting speed, level I for load for finish and level II for no of strokes which are indicated in the above table.

Employing design of experiments, are important statistical tools for designing high quality systems at reduced cost. Statistical design of experiments is a procedure that allows quick, economical, and accurate evaluation of processes and products that depend upon several variables. It is found that the parameter design of the Taguchi method provides a simple, systematic, and efficient methodology for optimizing the process parameters.

\section{REFERENCES}

[1] Taguchi G, Konishi S ,Taguchi Methods, orthogonal arrays and linear graphs, tools for quality American supplier institute, American Supplier Institute; 1987.

[2] Rao, Ravella Sreenivas; C. Ganesh Kumar, R. Shetty Prakasham, Phil J. Hobbs, The Taguchi Methodology as a statistical tool for biotechnological applications: A critical appraisal, Biotechnology Journal 3 (4):510-523. 
[3] W.T. Foster, Basic Taguchi design of experiments, National Association of Industrial Technology Conference, Pittsburgh, PA, 2000

[4] Domnita Fratilia, Cristian Caizar, Application of Taguchi method to selection of optimal lubrication and cutting conditions in face milling of AlMg3, Journal of Cleaner Production 19 (2011) 640-645

[5] Effect Of Honing Process Parameters On Surface Quality Of Engine Cylinder Liners International Journal of Engineering Research \& Technology (IJERT) Vol. 2 Issue 4, April - 2013 ISSN: 2278-0181 Pankaj S. Chavan Prof. M. S. Harne

[6] Surface Roughness Influencing Process Parameters \& Modeling Techniques for Four Stroke Motor Bike Cylinder Liners during Honing: Review..International Journal of Mechanical \& Mechatronics Engineering IJMME-IJENS Vol:15 No:01 106 156901-3737-IJMME-IJENS @ February 2015 IJENS I J E N S

[7] International Journal of Mechanical and Production Engineering, ISSN: 2320-2092, Volume- 1, Issue5, Nov-2013 A Review Of Optimization Process Parameters On Honing Machine 45 A REVIEW OF OPTIMIZATION PROCESS PARAMETERS ON HONING MACHINE

[8] Byrne D. M. , S. Taguchi: “The Taguchi approach to parameter design, "Quality Progress, vol. 20 (12), pp. 19-26, (1987).

[9] Ealey Lance A.: "Quality by design Taguchi methods and US industry". 2nd ed. Sidney: Irwin professional publishing and ASI Press;.p. 189-207, (1994).

[10] Hafeez K., H. Rowland, Kanji, S.Iqbal: "Design optimization using ANOVA", Journal of Applied Statistics, vol. 29, issue 6, pages 895-906, (2002).

[11]Logothetis, N. and Wynn, H. P.: "Quality Through Design: Experimental Design, Off-line Quality Control, and Taguchi's Contributions”. Oxford University Press, Oxford Science Publications, (1989) Montgomery, D. C.: “Design and Analysis of Experiments,” ed. John Wily, New York, 1991. 